\title{
New Classical Potential For Accurate Simulation of Atomic Processes in Si
}

\section{Citation}

Kaxiras, Efthimios, and K. C. Pandey. New classical potential for accurate simulation of atomic processes in Si. Physical Review B 38, no. 17: 12736-12739.

\section{Published Version}

http://dx.doi.org/10.1103/PhysRevB.38.12736

\section{Permanent link}

http://nrs.harvard.edu/urn-3:HUL.InstRepos:2958647

\section{Terms of Use}

This article was downloaded from Harvard University's DASH repository, and is made available under the terms and conditions applicable to Other Posted Material, as set forth at http:// nrs.harvard.edu/urn-3:HUL.InstRepos:dash.current.terms-of-use\#LAA

\section{Share Your Story}

The Harvard community has made this article openly available.

Please share how this access benefits you. Submit a story.

\section{Accessibility}




\title{
New classical potential for accurate simulation of atomic processes in $\mathrm{Si}$
}

\author{
Efthimios Kaxiras and K. C. Pandey \\ IBM Research Division, Thomas J. Watson Research Center, Yorktown Heights, New York 10598
}

(Received 29 August 1988)

\begin{abstract}
In a critical evaluation, we show that existing classical potentials are not suitable for calculating the energy of realistic atomic processes in $\mathrm{Si}$. We present a new potential which is especially suited to simulate processes in the diamond lattice rather than in high-energy bulk structures of $\mathrm{Si}$. Our potential is based on a very large quantum-mechanical data base. It consists of two- and three-body terms with short-range separable forms, and reproduces accurately the energy surface for atomic exchange in Si. Thus, it is ideally suited for molecular dynamics simulations of atomic processes in $\mathrm{Si}$.
\end{abstract}

The structural properties and the dynamical behavior of materials at the microscopic atomic level constitute a large area with many basic, interesting and completely unexplored problems. How do solids melt? How do atoms diffuse and are defects necessary for diffusion? What is the structure of the amorphous and glassy states and how is it stabilized? These are some typical problems that have been attracting increasing attention. Classical potentials give an approximate, but extremely simple, means of addressing such issues. Partly due to technological interest in semiconductors, in recent years there have been numerous attempts at constructing a classical potential for a prototypical semiconductor: namely, $\mathrm{Si}$. However, as will be discussed below, a potential which reproduces the properties of all phases of $\mathrm{Si}$ remains elusive, although attempts at constructing such a potential for specific applications have met with limited success.

In this paper, we present a new potential for $\mathrm{Si}$ which we believe is reliable and accurate for simulation of a large class of atomic processes. This potential is based on two premises. First, that one cannot construct a potential which describes accurately both the high-coordination, high-energy crystal structures ( $\mathrm{fcc}$, bcc, hexagonal closepacked, etc.) and the local distortions of the diamond lattice. Thus, one has to ask what is the potential intended for, and tailor its characteristics accordingly. Here, we have focused on constructing a potential which reproduces accurately the energy cost for a wide range of local distortions from the tetrahedral coordination, including the breaking and formation of bonds, as well as the cohesive energy and bulk modulus of the diamond lattice, which is the lowest-energy crystal structure of $\mathrm{Si}$. Though this may seem as a restriction on the scope of the potential, it is not a serious limitation because it contains most of the low-energy, physically accessible configurations. The second premise consists of using a large quantummechanical data base for constructing the potential: namely, the energy surface of atomic exchange. ${ }^{1}$ Such an extensive date base, representing a realistic physical process, had not been available to previous attempts at constructing $\mathrm{Si}$ potentials. These attempts by necessity relied heavily on existing quantum-mechanical calculations for high-coordination bulk structures of $\mathrm{Si}$, which, as will be shown below, has proven insufficient. Some advantages of our potential include a simple and physically transparent functional form, and a small set of parameters (seven) which can be easily fitted to the quantum-mechanical results.

A unique opportunity to perform a critical evaluation of existing classical potentials is offered by the recent quantum-mechanical local-density-functional (LDF) calculations of Pandey. ${ }^{1}$ The new element in this work is that an energy surface is calculated for the entire phase space associated with arbitrary rotations of two rigidly bonded atoms in the diamond lattice environment of bulk Si. The range of bond-length and bond-angle distortions encountered in this process is very wide. In addition, the fact that no particular symmetry restrictions were imposed guarantees that the range of configurations will be representative of a very wide range of possible local distortions in a solid with overall fourfold coordination. Furthermore, there are particular paths in this phase space which correspond to physically important processes such as self-diffusion. Finally, although LDF calculations in general involve some uncertainty due to convergence limits, the energy surface under consideration was obtained with exhaustive parameters and should be highly reliable (a detailed discussion of convergence is given in Ref. 1). All these features of the multidimensional energy surface for atomic exchange conspire to make it an ideal "proving ground" for classical potentials.

The simplest way to compare the predictions of classical potentials for the energy surface of atomic exchange is in terms of a specific phase-space path. For this comparison, we have chosen the concerted exchange (CE) path ${ }^{1}$ which is important for several reasons: it is the path of lowest energy for a complete exchange of two bulk atoms; it spans a wide subset of the radial and angular distortions encountered in the entire phase space of the atomic exchange; and it determines the activation energy for selfdiffusion through atomic exchange in $\mathrm{Si}$. During the $\mathrm{CE}$, a pair of atoms start from the equilibrium configuration and rotate around their center of mass until they exchange positions. The motion can be described in terms of the polar angle $\theta$ which varies from $0^{\circ}$ to $180^{\circ}$ and the azimuthal angle $\phi$ which at each stage is a function of $\theta$. When the system departs from the equilibrium configuration $\theta$ $=0^{\circ}$ (which defines the zero of energy) the energy starts 
to increase as bonds are being distorted to the point of breaking, while other bonds are formed. The energy reaches a maximum value at $\theta=90^{\circ}$, which is the saddlepoint configuration (see also Ref. 1).

We have chosen to examine the most established among existing potentials, including the potentials of Stillinger and Weber, ${ }^{2}$ Biswas and Hamann, ${ }^{3,4}$ Tersoff, ${ }^{5}$ and Dodson. ${ }^{6}$ The potential labeled "new" Biswas and Hamann ${ }^{4}$ is a variation of the "old" Biswas and Hamann potential ${ }^{3}$ designed to improve the defect formation energies in $\mathrm{Si}$. The Dodson potential, ${ }^{6}$ a variation of the Tersoff potential, ${ }^{5}$ was introduced to fix the incorrect prediction that the diamond lattice is not the lowest-energy crystal structure of $\mathrm{Si}$. Our calculations for the energy of the $\mathrm{CE}$ path using the above potentials are shown in Fig. 1, together with the quantum-mechanical results of Pandey. ${ }^{1}$ As seen from Fig. 1, these potentials are qualitatively inadequate in three aspects with important physical consequences: they exhibit unrealistic structure (local minima), they fail to reproduce the activation energy, and they have wrong curvature.

The first important shortcoming concerns the existence of several local minima in the classical energy curves which are absent from the LDF results. This feature is more pronounced in the energy curves of the Tersoff and Dodson potentials. A direct consequence of the local minima is the prediction of erroneous metastable configurations which may give rise to unphysical structures, for example in grain boundaries and amorphous materials. To illustrate this point, we shall discuss in some detail one particular metastable configuration among the several predicted by the potentials, which has played an important role in the literature. This configuration corresponds to $\theta=60^{\circ}$ or $120^{\circ}$ in the CE path (the two configurations are structurally equivalent). It is interesting that this configuration is predicted to be metastable by all of the potentials considered in Fig. 1 except for the Tersoff potential (which exhibits several other deep metastable structures). By contrast, there is no local minimum in the LDF results throughout the CE path. This means that the system, once it reaches the $\theta=60^{\circ}\left(120^{\circ}\right)$ configuration, will evolve without encountering any energy barrier to the equilibrium $\theta=0^{\circ}\left(180^{\circ}\right)$ configuration, contrary to what the classical potentials would predict. Stillinger and Weber, for example observed a metastable defect in their molecular-dynamics simulation ${ }^{2}$ which, neglecting relaxations, corresponds structurally to the $\theta=120^{\circ}$ configuration of the CE (see Fig. 13 of Ref. 2). The effect of relaxation will be to lower the energy of this configuration, both in LDF and in classical potential calculations. It is not clear to what degree the relaxed configurations are metastable. The interesting aspect of the comparison is that the classical potentials give a qualitatively wrong description for the unrelaxed configuration which clearly is not metastable according to LDF. The observation of this metastable defect by Stillinger and Weber was used as evidence in support of the so-called "sillium" model for amorphous silicon, ${ }^{7}$ which is based on repeatedly distorting crystalline silicon by creating $\theta=60^{\circ}$ or $120^{\circ}$ defects and allowing the structure to relax. Thus, the potentials considered in Fig. 1 may give rise to irregular structures whose relative stability is due to unrealistic features in the potential. The abundance of such structures is directly related to their energy, and the resulting statistics in models of amorphous Si may be misleading.

A second serious shortcoming is that the activation energy of the $\mathrm{CE}$ (corresponding to the saddle-point configuration at $\theta=90^{\circ}$ ) as given by the various potentials differs from the LDF results by $1-3 \mathrm{eV}$. For some of the potentials the $\theta=90^{\circ}$ configuration is not a saddlepoint in the energy surface (i.e., a total maximum along the CE path), but a local minimum. Finally, the third important shortcoming concerns the curvature of the classical energies which deviates considerably from the curvature of the LDF energy. This feature is particularly important for the dynamics of atoms, such as the diffusion rate for example, which involves integrations of the exponentiated energy around the equilibrium $\left(\theta=0^{\circ}\right)$ and the saddle-point $\left(\theta=90^{\circ}\right)$ regions. The curvatures of the existing potentials (see Fig. 1) would give unreasonable estimates for the diffusion rate, with the possible exception of the "new" Biswas and Hamann potential. In fact,

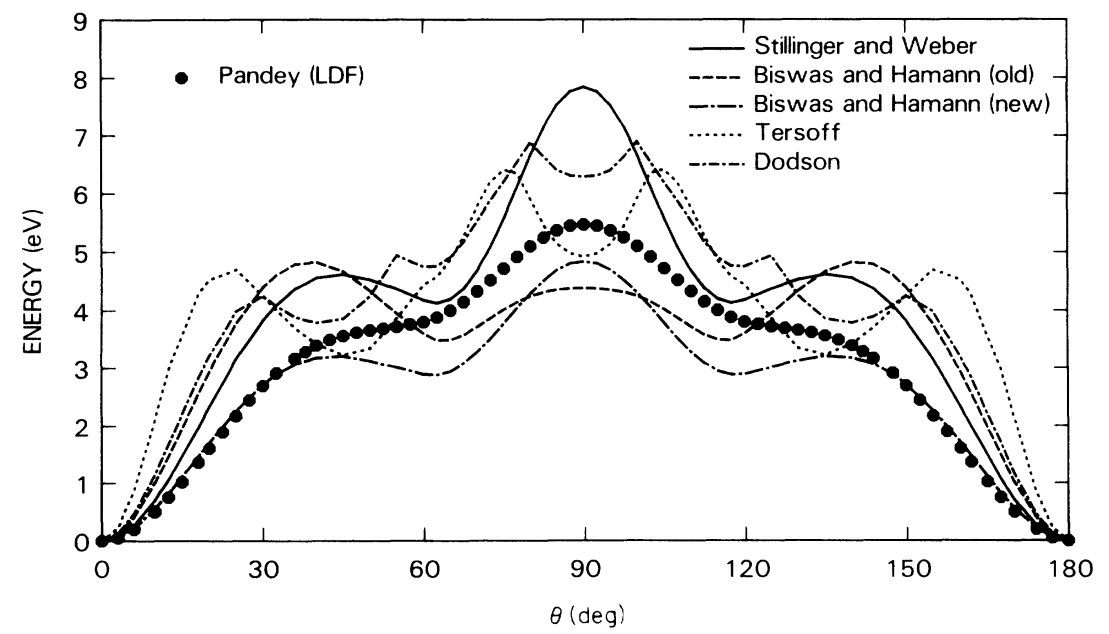

FIG. 1. The energy of the concerted exchange path as given by the various classical potentials and by LDF calculations. 
this potential was the only one, among the potentials considered here, to incorporate in the data base the energies of local distortions of the diamond lattice. The fact that it gives a reasonable overall description for the energy of the CE path (see Fig. 1) shows how important the energies of local distortions are as opposed to the energies of highcoordination bulk structures.

The potential proposed in the present work reproduces the energy of the CE path to better than $0.1 \mathrm{eV}$ (it is almost indistinguishable from the LDF results on the scale of Fig. 1), and has, therefore, distinct advantages over other potentials. We describe briefly the important features of our potential, beginning with the two-body term which, after a search over different functional forms, was chosen to be the difference of two Gaussians:

$$
V_{i j}=A_{1} \exp \left(-\alpha_{1} r_{i j}^{2}\right)-A_{2} \exp \left(-\alpha_{2} r_{i j}^{2}\right)
$$

( $r_{i j}$ is the distance between atoms $i$ and $j$ ). This form is similar to the one used in the "new" Biswas and Hamann two-body term. ${ }^{4}$ The values of the parameters are $A_{1}$ $=57.316072, \quad A_{2}=6.4373054, \quad \alpha_{1}=0.82335230$, and $\alpha_{2}=0.19061589$ (lengths are in $\AA$ and energies in eV). The two-body term is short ranged, reaching zero at the cutoff radius $r_{c}=5.5 \AA$, without the use of any cutoff function.

The three-body term contains the dependence of the energy on the angle $\theta$ between two bonds and a simultaneous dependence on the two bond-lengths subtending $\theta$. The radial dependence of the three-body term was chosen to be a Gaussian by analogy to the two-body term. The angular dependence, again after a search over different functional forms, was chosen to be of the generalized Keating-form, that is a power expansion in the binomial $g(\theta)=\left(\cos \theta+\frac{1}{3}\right)$ which vanishes at the tetrahedral angle. The full three-body potential is a symmetrized sum over all triplets of atoms $i j k, V_{3}=V_{i j k}+V_{j k i}+V_{k i j}$, with

$$
V_{i j k}=\exp \left[-\beta\left(r_{i j}^{2}+r_{i k}^{2}\right)\right]\left[B g^{2}(\theta)+D g^{4}(\theta)\right],
$$

where $\theta$ is the angle at the $i$ vertex of the $i j k$ triangle, subtended by the bonds $r_{i j}$ and $r_{i k}$. The above form of the three-body potential is separable (it allows the reduction of the three-body sums to two-body sums ${ }^{3}$ ). This property is important for practical reasons, because it reduces significantly the amount of computational effort. The values of the parameters of the three-body term are $B$ $=10.188418, D=-9.113678$, and $\beta=0.18642554$. The three-body term also vanishes for bond lengths larger than the cutoff radius $r_{c}$.

We shall now consider some of the predictions of our potential for structures other than those used in fitting the parameters. Specifically, we shall discuss formation energies for point defects and cohesive-energy curves for various bulk structures. The most common point defects in $\mathrm{Si}$, which have been extensively studied in the literature, are vacancies $(V)$ and interstitials which can exist in two high-symmetry configurations: the tetrahedral $(T)$ and the hexagonal $(H)$. Based on our potential, the formation energies of these defects in unrelaxed configurations are $E_{V}=2.9 \mathrm{eV}, E_{T}=2.7 \mathrm{eV}$, and $E_{H}=4.6 \mathrm{eV}$. Previous LDF calculations ${ }^{8}$ have reported somewhat larger values with large error bars $\left(E_{V}=5-6, E_{T}=5-7\right.$, and $E_{H}=6-8$ $\mathrm{eV}$ ). However, recent and more accurate LDF calculations give lower formation energies, ${ }^{9}$ which are in fact very close (within $1 \mathrm{eV}$ ) to the energies obtained from our potential. By contrast, the formation energies obtained from other potentials differ from the LDF results by

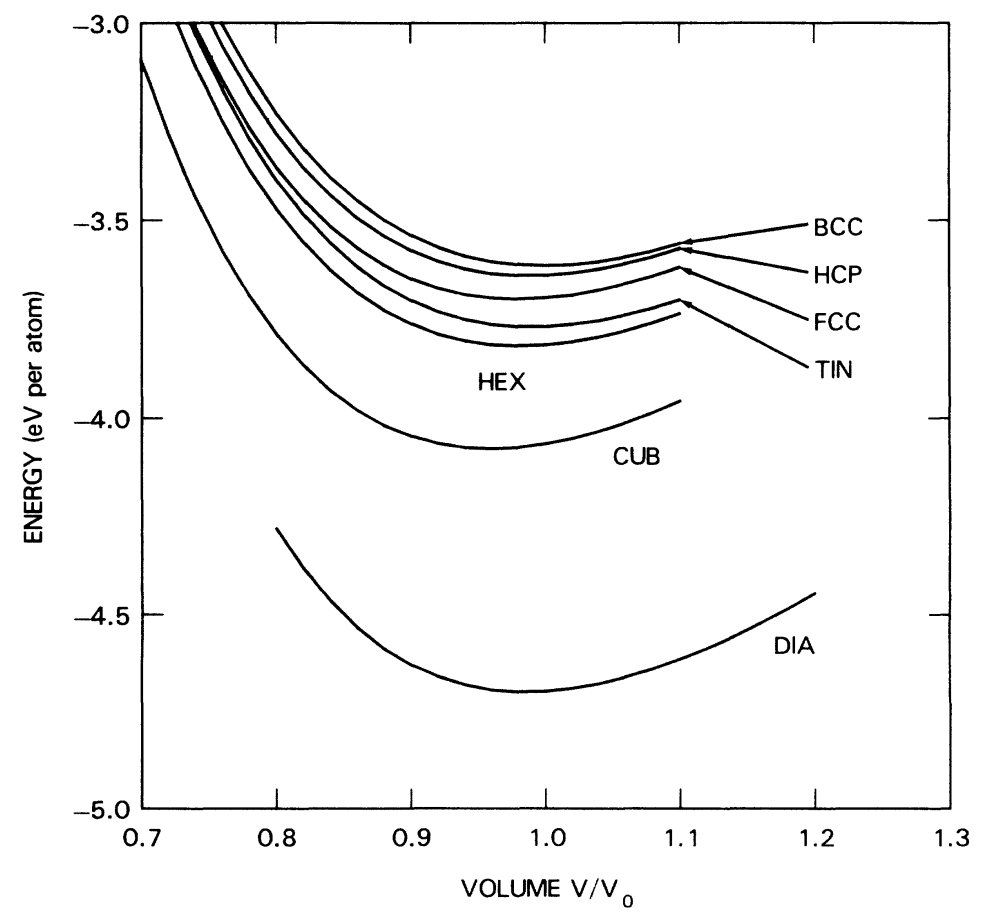

FIG. 2. Cohesive energy per atom vs atomic volume (normalized to the experimental atomic volume $V_{0}$ of diamond), for various bulk structures of $\mathrm{Si}$ as given by our classical potential. 
several $\mathrm{eV}$ in some cases. The most realistic estimates for $E_{V}$ and $E_{T}$ are obtained using the "new "Biswas and Hamann potential ${ }^{4}$ or the Tersoff potential ${ }^{5}$ (both potentials, however, give inaccurate estimates of $E_{H}$ ).

For a second test of our potential we consider the cohesive-energy vs atomic-volume curves for bulk structures of $\mathrm{Si}$ which exhibit high coordination. ${ }^{10}$ This stringent test of the potential actually lies outside the domain of its validity since it was tailored to describe accurately local deviations from the tetrahedral coordination. Thus, we do not expect good agreement with LDF calculations. The predictions of our potential are shown in Fig. 2. The LDF energy curves for the same bulk structures have minima roughly at $0.8 V_{0}$ (where $V_{0}$ is the experimental atomic volume of diamond) rather than at $0.95 V_{0}$ as predicted by our potential, and they vary over a much smaller energy scale than what is shown in Fig. 2 (see, e.g., Fig. 1 of Ref. 4). One important feature is well reproduced, however: namely, that diamond is the crystal lattice of lowest energy. The minimum and the curvature of our classical cohesive-energy curve for the diamond lattice are by construction equal to LDF results. Interestingly, most of the other potentials give poor estimates for the relative energies of bulk structures, except for the "old" Biswas and Hamann potential which was specifically constructed to fit the bulk-structure curves. ${ }^{3,4}$

The difficulty in reproducing the energies of several bulk structures as well as the energy of point defects in $\mathrm{Si}$, using the same potential, has been noted previously. ${ }^{4}$ In view of this limitation, we have concentrated here in constructing a potential that reproduces with sufficient accuracy the energy cost for a wide range of local distortions, including the breaking and formation of bonds, in the diamond lattice. We have shown that our potential is far more reliable than the existing potentials in studies of problems where fourfold coordination is predominant. As with all classical potentials, however, it will not be possible to capture with our potential the physics in situations where different charge states or other electronic excitations are important. It would also be unrealistic to use this potential for problems which are far removed from its region of validity, as, for example, in modeling the molten state where the average coordination is higher than four. On the other hand, this potential is particularly well suited for studies of amorphous structures obtained by quenching the melt, in which case the coordination in the melt is not important, but the final coordination in the amorphous network is crucial. Other possible applications include molecular-dynamics simulation of diffusion and modeling of extended defects such as dislocations and grain boundaries.

We wish to acknowledge helpful discussions with J. D. Joannopoulos and thank N. D. Lang and E. Pytte for useful comments on the manuscript.
${ }^{1}$ K. C. Pandey, Phys. Rev. Lett. 57, 2287 (1986).

${ }^{2}$ F. H. Stillinger and T. A. Weber, Phys. Rev. B 31, 5262, (1985).

${ }^{3}$ R. Biswas and D. R. Hamann, Phys. Rev. Lett. 55, 2001 (1985).

${ }^{4}$ R. Biswas and D. R. Hamann, Phys. Rev. B 36, 6434 (1987).

${ }^{5}$ J. Tersoff, Phys. Rev. Lett. 56, 632 (1986).

${ }^{6}$ B. W. Dodson, Phys. Rev. B 35, 2795 (1987).

${ }^{7}$ F. Wooten, K. Winer, and D. Wearie, Phys. Rev. Lett. 54, 1392
(1985); F. Wooten and D. Wearie, Solid State Physics, edited by $\mathbf{H}$. Ehrenreich and D. Turnbull, (Academic, Orlando, 1987), Vol. 40, p. 1.

${ }^{8}$ G. A. Baraff and M. Schluter, Phys. Rev. B 30, 3460 (1984); Y. Bar-Yam and J. D. Joannopoulos, Phys. Rev. Lett. 52, 1129 (1984); R. Car, P. J. Kelly, A. Oshiyama, and S. T. Pantelides, ibid. 52, 1814 (1984).

${ }^{9}$ K. C. Pandey (unpublished).

${ }^{10}$ For these calculations $r_{c}$ was scaled with the atomic volume. 